\title{
ISLAMIC MEDICINE IN NORTH AMERICA
}

\author{
By Ahmed Elkadi, M.D. \\ Akbar Clinic, 4000 East 3rd Street, Panama City, Florida 32401 \\ Presented at the Spring Convention of IMA. Orlandn, Florida, March 17, 1984
}

\section{DOI: http://dx.doi.org/10.5915/16-1-5956}

Lslamic Medicine as a comprehensive health care system does not exist in North America, nor anywhere in the world for that matter. We have the concept of Islamic Medicine. We have the plan to establish it. But we do not have it yet. We have been talking about Islamic Medicine for the past 16 years. During the 16 th annual convention of IMA in 1983, the milestones asd achicvements made in the field of Islamic Medicine as of 1983 were reviewed. ${ }^{2}$ This showed that the concept and definition are already formulated, the plan worked out, the organizational stmucture formod, and a network of communication put into gear 'Th.esce are all essential preparatory steps, hut the main bulk of work is still to be done.

It is like erecting a huge building. First, it has to be decided as to what type of building it is going to be and what function it will serve. Then a name has to be given to it, whether it is an apartment complex, condominiums, office building, Moscuse, convention center or the like. Next, comes the fersibility study. then the selection of an architect and a builder, then the drawing of the blueprints, with possible correction and modifications, then the allocation of finances and building materials, as well as the selection of a site. Finally, the various permits and licenses have to be obt ained and the legal requircments have to be met. In between, there will be meetings, hearings, and conferences to be sure that everything is available. Then, and only then, can the actual construction work be started.

This is wherc we are now with regard to Islamic Medicine. We have passed through all the essential preparatory steps and are now ready to start the actual construction work. The value of these preparatory steps, however, should not be underestimated. It is of the utmost importance to have a clear vision and proper orientation right from the start. In such a long road leading to our objectives, which may take anywhere from 20 to 50 years to readize, a start which is even just a few degroes off the proper direction will lead us, after 20 or 50 years, to a destination which is quite different and far from the one we had intended. Any time or effort spent in the proper preparation is an investment well spent.

Now, we have a definition of Islamic Medicine which is:

"The most up-to-date medical science and technology combined and conforming with the Divine teachings of Islam." \&

Wc also have the six basic features and criteria of
Lslamic Mexdicine outlined. These are: Being excellent and superior, having faith and Divine ethics, being oricnted, beina commehensive, being universal. and last, lut not 1 ' st, being scientific.' 2 We have the fea ". .ir sudies which show us that Islamic Medicine is not only teasible but also badly needed as a superior alternative to the existing brands of the healing arts. ${ }^{3}$ 4 We also have the vast Muslim resources, intellectual as well as material, waiting to be pooled and utilized. Adding to this the existing, reasonably well-developed organizational structures and networks of communication at national and international levels, it appesars that we are in a position to start the actual construction work.

At this level, let us see what a comprehensive health care system should entajl. It should have three basic components. It should have facilities for education and trainjing with available, properly prepared curricula and teachers. It should have facilities for rendering health care services be they preventive, diagnostic or therapeutic. And it should have an ongoing research program covering all aspects of research be it clinical or experimental, whether applicable to humans or animals. The quality and level of performance of these three components will determine the quality and perfornance of the final product. 'To date, there are several such components of one type or another, or just parts thereof, being developsed and established in various parts of the world. Here, in North America, we should be proud and thankful to God the Almighty for being in a position to witness the birth of a unique facility which will hopefully present all three components combined. By this, I mean the Akbar Clinic which houses the Akbar Institute of Health. The physical facilities of the Akbar Clinic are currently being completed and should be ready for operation within six to eight weeks insha Allah. Like any newbom, it is still weak, vulnerable and not yet able to show its full-fledged potential. However, it has the genetic setup and composition which will determine its personality and potentials. Given time and proper care and nurturing, it will insha Allah grow and develop to its planned format of an lsiamic medical Institution providing education, rexearch and health carc services.

Knowing the magnitude of a monumental undertaking such as the total Islamization of the health sciences and the establishment of a new breed of the healing arts, it is obvious that the output of only one unit is nowhere near enough. It will take the combined efforts of several such units in North America and all over the world. However, the experience at Akbar 
could be looked upon as a possible leading prototype for these units. The very preliminary working plans I am going to share with you in the next few minutes are only a very simple and crucle example of what could be done.

It should go without saying that the properly oriented curricula suitable for the teaching of Islamic Medicine cannot be formulated just by sitting in the library or study chamber. They have to be based on the experiences and findings of long years of resenrch and practice. This may lead to the conclusion that medical education at Akbar will have to wait for many long years before it can be a reality. While this is true with regatrd to a comprehensive Curriculum for a medical school. some type of medical education is needed and is actually going on right now. Continued medical education for the staff members of Akbar is an essential ongoing activity at present. This takes place in the form of bi-weckly preparation and presentation of modical subjects in the various specialties, as well as a review of specialty journal material. Initially, we thought that this would be the extent of our nedical educational activities for years to come. However. and due to certain favorable circumstances, we are blessed with a small number of medical students who rotate at Akbar to gain part of their clinical experience and share with us our developmental stage. The presence of medical students at Akbar has a great educational value for the staff members who need someone to help them develop and practice their teching skills. Those of us involved in teaching should realize how much learning it takes a teacher to do a good tcaching job. So much for the educational aspect of Akbar at this stage of its development.

The next component of a comprehensive health care srstem is, as mentioned above; the heal th care services. Right from the beginning, the Akbar Clinic will provide the comprehensive diagnostic services usually found only in a hospital setup although the Clinic will start as an ambulatory facility. This will be supported by a sophisticated home health care service, in addition to the ability to keep patients up to 24 hours at the Cinic facility. We hope that, within the first year or two, we shall be able to have some type of lodging facility attached to the Clinic although this may not necessarily he labeled a hospital. It may take the form of an extended care unit, a drug rehabilitation unit, or cven just a Clinic Inn, depending on the legal approvals and the number of beds approved by state health agencies. The ultimate goal is to have a complete self-sufficient hospital facility as part of the Akbar facility. Knowing the existing legal and political situation, this goal may take several years to be achieved. However, unexpected circumstances may take place that will allow the earlier realization of this objective. Until then, we shall be using the local hospital facilities in town to satisfy our inpationts' needs who absolutely require a real hospital setup.
Last but not least, is the research component which, I feel. is the basic foundation of any quality health care system. Without ongoing research, there will be stagnation of knowtedge, and stagnation leads to decay. Since the priorities and orientation of Islamic medicine are in some or many aspects different from those of traditional contenuporary medicine, there is no substitute for our own reacuarch programs which have to be tailored to our neeck and criteria. At this very early stage of ous development and considering our very' limited resources, we have a few humble research itens on our agenda. Here I should like to clarify that I shall be limiting my comments to research topics other than just literature review and simple case reports which could and should be done by anyone with interest and a reasonable access to a library. The research subjects I shall be talking about are the ones which require an institution to be conducted in. Even the ones which appear to be simple and straightforward still need a facility where the data, clinical or experimental, can be collected. From the nature of some of the topics which I shall be mentioning, it will become clear why the facility noeds to be ours and/or under our enntrol.

One area of interest is the area of evaluation and documentation of the physiologic effects of the Quran in mar. Claims are made of the healing power of the Quran. The question is how, how much, and why it works. This area of research does not al all fall in the field of parapsychology or mysterious spiritualism. It deals with the real physiologic and pathophysiologic responses of the human body' and their relations to discase processes. There is enough evidence to indicate that stress in various forms, mental or physical, leads to impaired immunity either through the cortisol secretion or any other neuro-endocrine meclianism. Impaired immunity opens the door to all types and varieties of somatic disorders, many of them are quite fatal. The Quran is expected to have some stressreducing effect, and consequently may, and should prohably have some favorable effect on the immune responses of the host. The question again is how, how much, and why it works. Another question is whether different types of Quaranic verses have different effects on the body. A third question is the effect of the language of the Quran and its translations, and the type of faith of the listening subjects. Examining all these variables under a randomized and controlled setup, and comparing the Quran to a variety of other streas-reducing modalities, as well as placebos, produces quite an involved study protocol which will require a series of well-planned studies. The documentation of the effects will utilize a variety of techniques ranging from documentation of gross physiologic responses using Biofeedback techniques to sophisticated measurements of cellular and humoral responses of the immune system.

Another area of research is in the field of treatment 
of cancer using a multi-modality, specific and nonspecific immunotherapy approach. This will be done in conjurnction with Dr. Issels" Institute of Applied Oncology in West Germany. Astesunding and almost unbelievable results have been achieved at Dr. Issels" facility in the treatment of advanced cancer cases over the last 20 years. However. the method has not been scientifically evaluated. It is also looked upon as unconventional, and many researchers are hesitant to try such a polypragmatic approach. In conventional immunotherapy research, only one modality is evaluated at a time. This may be the main reason why not much success has been achieved with conventional single modality immunutherapy. At Akbar, we plan to evaluate the clinical results of Dr. Issels approach following prospective randomized controlled study techniques. Besides, we plan to evaluate the collular and humoral responses of the immune system to the therapy. The same multi-modality imunotherapy may also be effective in the treatment of various chronic benign conditions which will have to be evaluated through separate study protocols.

A third area of interest is in the field of pharmaceutical preparations. One part of the research will be towards the development of an alcohol-free pharmacopeia.

Another part of the pharmaceutical research is the evaluation of the effects of some of the herbs and plant products which have been used for medicinal purposes for hundreds of years. This will require a multitude of clinical and pharmacological studies. This work will complement some of the work currently being done at some facilities in Kuwait and India.

Along the line of pharmaceutical research, there may also be some limited pharmaceutical industry for the production and marketing of some Akbar Brand of medicinal preparations.

A fourth area of research which we have on our preliminary agenda is the evaluation of Acupuncture anesthesia and analgesia in surgical patients during the intra-operalive and yost-operative phases of treatment, comparing its results with those of conventional methods.

Other topics on the list include the evaluation of various nutritional approaches and rehabilitative methods.

Looking upon the above-mentioned research areas, the question may be raised as to what relation these have to Islamic medicine. Altbough the listed topics are varied and deal with unrelated subjects, they all have something to do with one or more of the six basic features and criteria of Islamic Medicine. They either deal with the search for a superior alternative, be it a more effective or a safer modality of treatment; or the development of a treatment system which is in compliance with Divine ethics; or providing a coniprehensive treatment modality that takes care of the physical as well as the spiritual needs; or showing a universal open-minded attitude willing to investigate some form of treatment which is ancient, exotic or, to say the least, unconventional and where - regardless of the resenrch arca or subject of specialty - all matters are subjected to scientific cvaluation and scrutiny.

Another question may be raised as to how this research activity will be financed. Akbar has not used any grants or donations for the establishment of its facilities. All the finarcing was obtained fron the Islamic Bank in Luxembourg on a lease-purchase basis. The money will be paid back from the revenues of the Akbar Clinic insha Allah. However, we shall be soliciting grants for the various research studies we plan to undertake. But, if worse comes to worse and grants cannot be obtained, the Akbar Clinic will try to finance as many research activities as possible, depending on its own revenues. If we have to depend completely on our own internal resources, the research acitivities will have to be somewhat linited, at least to start with.

With this brief presentation, I have submitted to you a sample of our actual working plan at Akbar which will have to be expanded and probably' modified over the nexl few years. I hope that this humble start will provide a useful part of the North American contribution towards the establishment of Islamic Medicine.

Bibliography
1. Elkadi, A.: What is Islamic Merlicine? Presented at the First International Conference on Islamic Medicine, Kuwait, January 1981, and published in Proctedings of same.

2. Elkadi, A.: Islamic Medicine 1983: Milestones. Presented at 16th Annual Convention of IMA of North America, Chicago, September 1983.

3. Elkadi, A. and Ghoor, A.: Correlation between Islamic Values and the Incidence of Cancer. Presented at the 14th Anmual Convention of IMA of North America, Orlando. December 1981, and published in Proceedings of Second International Conference on Islamic Medicine in Kuwait, March 1982.

4. Elkadi, A.: Correlation between Islamic Values and the Incidence of Disease. Presented at the 3rd Annual Convention of IMA of South Africa, Lenasia, May 1983. 